\title{
Markers for oxidative stress in the synovial fluid of Thoroughbred horses with carpal bone fracture
}

\author{
Nao TSUZUKI $^{1^{*}}$, Yoshinori KANBAYASHI ${ }^{2}$ and Kanichi KUSANO ${ }^{2}$ \\ ${ }^{1}$ Obihiro University of Agriculture and Veterinary Medicine, Hokkaido 080-8555, Japan \\ ${ }^{2}$ Racehorse Hospital, Miho Training Center, Japan Racing Association, Ibaraki 300-0493, Japan
}

\begin{abstract}
Arthritis is thought to cause oxidative stress in synovial fluid in humans, but there have been few reports in horses. To evaluate oxidative stress in synovial fluid in horses, this study used 19 horses with unilateral fracture of the carpal joint bone. Synovial fluid was collected from the carpal joint on the fracture (arthritis group) and contralateral (control group) sides. Diacron-reactive oxygen metabolites (d-ROMs) and biological antioxidant potential (BAP) were then measured, and the oxidative stress index (OSI) was calculated. $d$-ROMs and OSI of the arthritis group were significantly higher than the control group. $B A P$ of the arthritis group was significantly lower than the control group. Thus, this study revealed that oxidative stress develops in the synovial fluid of horses during arthritis.
\end{abstract}

Key words: arthritis, horse, oxidative stress, synovial fluid

\author{
J. Equine Sci. \\ Vol. 30, No. 1 \\ pp. 13-16, 2019
}

Oxidative stress is defined as the state in which the concentration of reactive oxygen species (ROS) exceeds that of antioxidants $[12,13]$. Although ROS are removed by antioxidants, complete removal is not possible in the case of excessive ROS production. The residual ROS causes cell damage through oxidative damage to the cell membrane and DNA and protein denaturation [13]. Inflammation activates neutrophils and induces the release of ROS from neutrophils $[2,8]$. Also, arachidonic acid generated during inflammation induces ROS production [4]. Thus, it is considered that oxidative stress can occur at the inflammation site characterized by excessive ROS production. In synovial fluid during arthritis, oxidative stress may be present.

In humans, oxidative stress may be an exacerbating factor for joint diseases, such as temporomandibular joint disease $[1,9,10]$. It has been proved that cultured chondrocytes provide weak defense against oxidative stress and are killed when exposed to ROS [15]. Therefore, articular cartilage is a weak tissue against oxidative stress. Oxidative stress in synovial fluid is thought to promote the destruction of articular cartilage. Therefore, evalua-

Received: February 19, 2018

Accepted: November 26, 2018

*Corresponding author. e-mail: tsuzuki@obihiro.ac.jp

(C)2019 Japanese Society of Equine Science

This is an open-access article distributed under the terms of the Creative Commons Attribution Non-Commercial No Derivatives (by-nc-nd) License. (CC-BY-NC-ND 4.0: https://creativecommons.org/licenses/ by-nc-nd/4.0/) tion of oxidative stress in synovial fluid is important for assessment of severity of arthritis [1]. However, only a few studies have reported oxidative stress in the synovial fluid of horses during arthritis [5, 17, 21]. Furthermore, all these reports performed comparisons among separate horses, and there are no reports that have compared oxidative stress in synovial fluid of joints with arthritis and synovial fluid of healthy joints in the same horse. Since diet and exercise level affect oxidative stress, evaluation with the same individual is important for evaluating whether oxidative stress is occurring [22].

For these reasons, we collected synovial fluid at the time of fracture of the carpal joint bone, which causes post-traumatic osteoarthritis (a form of arthritis) in horses, and evaluated the oxidative stress in the synovial fluid. For comparison with synovial fluid from a healthy joint, we used unilateral fractures, in which synovial fluid from the nonfractured side was collected and used as a control. This study is the first to compare oxidative stress in joints of the same horse.

This experiment was approved by the Animal Care and Use Committee at the Racehorse Hospital, Miho Training Center, of the Japan Racing Association. Nineteen racehorses (11 males, 7 females, 1 gelding; mean $\pm \mathrm{SD}, 3.2 \pm 0.6$ years) with fracture of the carpal joint bone (either carpal bone and/or distal end of the radius) were used, and the bone fragment was removed by performing an arthroscopic surgery. Unilateral fracture was confirmed by lameness examination and X-ray examination for both forelimbs. The fracture side 
was right in 13 cases and left in 6 cases. The fractures sites were the distal end of radius $(\mathrm{n}=14)$, radial carpal bone $(n=2)$, intermediate carpal bone $(n=1)$, distal end of radius and intermediate carpal bone $(\mathrm{n}=1)$, and third carpal bone $(n=1)$. In addition, it was confirmed that arthritis was present in the fractured joint and not in the contralateral joint on physical examination (confirmation of heat and swelling by palpation). The median days from fracture to surgery were 7 days ( 3 to 12 days). All horses were administered nonsteroidal anti-inflammatory drugs (Diclofenac, $1 \mathrm{mg} / \mathrm{kg}$, p.o.; Diclofenac Na, Sawai Pharmaceutical, Osaka, Japan) until the day before surgery. Arthroscopy was performed under general inhaled anesthesia for each horse. Synovial fluid samples $(1 \mathrm{~m} l)$ were collected from both carpal joints just before the surgical incision was made. The synovial fluid from the fractured side was included in the arthritis group, whereas that from the nonfractured side was included in the control group. Samples were stored at $-80^{\circ} \mathrm{C}$ until used for further assays. After sample collection, diacron-reactive oxygen metabolites (d-ROMs) and biological antioxidant potential (BAP) were measured using a free radical analyzer (Free Carpe Diem, Diacron International, Grosseto, Italy). We calculated the oxidative stress index (OSI) from the measured d-ROMs and BAP $(\mathrm{OSI}=\mathrm{d}-\mathrm{ROMs} / \mathrm{BAP} \times 100)$ $[3,14,18]$.

d-ROMs, BAP, and OSI were normally distributed; therefore, they were expressed as mean $\pm \mathrm{SD}$. All data were analyzed using Statcel3 (OMS Publishing Inc., Tokorozawa, Japan). Differences between the arthritis group and control group were determined using the paired $t$-test. The significance level was set at $P<0.05$ for all analyses.

Joint swelling and heat were confirmed in the affected joints in all horses. d-ROMs, BAP, and OSI are shown in Fig. 1a-c, respectively. d-ROMs in the arthritis group showed significantly higher levels than those in the control group $(P=0.02)$. BAP in the arthritis group showed significantly lower levels than those in the control group
( $P=0.0002)$. OSI in the arthritis group showed significantly higher levels than those in the control group $(P=0.0002)$.

High d-ROMs values indicate higher production of ROS, and high BAP values indicate higher antioxidant capacity. Celi et al. reported d-ROMs and BAP as simple and precise parameters for assessing oxidative stress in horses [3]. d-ROMs and BAP can be easily measured, so it is considered possible to apply them clinical situations [3]. Oxidative stress develops when the balance between ROS and antioxidants favors ROS production. Therefore, it is also important to evaluate the grade of oxidative stress using the OSI, which indicates the balance between d-ROMs and BAP. High OSI values indicate higher oxidative stress. In previous reports, $\mathrm{d}$-ROMs and BAP were mainly measured using serum or plasma samples [3], and there were no reports of measurements in synovial fluid. However, synovial fluid is a mixture of filtrate of plasma and hyaluronic acid and glycoprotein synthesized by synoviocytes. Therefore, since d-ROMs and BAP can be measured them with plasma, it was considered possible to measure with synovial fluid.

In this study, significantly higher d-ROMs values, significantly lower BAP values, and significantly higher OSI values were confirmed in the arthritis group than those in the control group. Because a significant increase in OSI was confirmed in the arthritis group, we considered that oxidative stress was generated in the synovial fluid on the fractured side. In the arthritis group, a significant increase in the d-ROMs and a significant decrease in BAP were observed. Therefore, the increase in OSI can be attributed to both excessive generation of ROS (i.e., increase in d-ROMs) and consumption of antioxidants (i.e., decrease in BAP).

Small amounts of ROS are quickly eliminated by antioxidants. Thus, when small amounts of ROS are generated, only a decrease in antioxidant capacity occurs [20]. In this study, a significant increase in d-ROMs on the fractured side was confirmed. Therefore, it was thought that excessive ROS were generated in the joint fluid on the fractured side, (a)

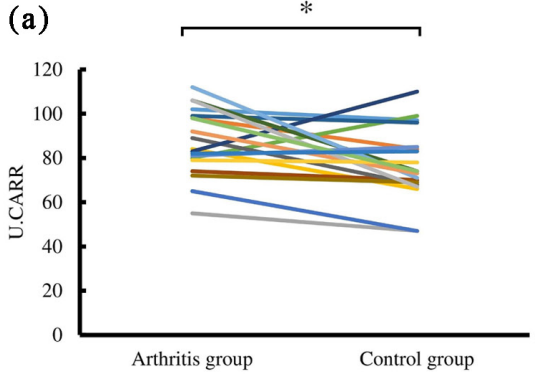

(b)

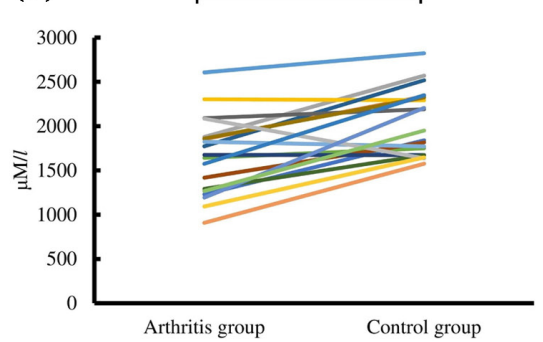

(c)

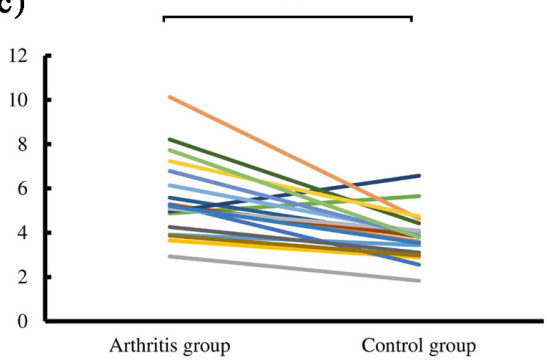

Fig. 1. d-ROMs, BAP, and OSI are shown in a-c, respectively. a: The arthritis group showed a significantly higher d-ROM level (87.2 \pm 15.0 U.CARR) than that the control group (76.6 \pm 16.3 U.CARR). b: The arthritis group showed a significantly lower BAP (1,661.5 \pm $440.3 \mu \mathrm{M} / l)$ than the control group $(2,050.0 \pm 377.0 \mu \mathrm{M} / l)$. c: The arthritis group showed a significantly higher OSI (5.6 \pm 1.8$)$ than the control group $(3.9 \pm 1.1) . * P<0.05 ; * * P<0.001$. 
which could not be eliminated by antioxidants. Cultured chondrocytes are weak for oxidative stress and are killed when exposed to ROS [15]. Therefore, damage to articular cartilage present at sites other than the injured site may occur due to the presence of ROS. Articular cartilage other than that at the injured site may be damaged by oxidative stress in synovial fluid. Therefore, reducing oxidative stress in synovial fluid may contribute to the preservation of the articular cartilage.

This study revealed that oxidative stress develops in synovial fluid during arthritis due to carpal joint bone fracture. In horses, there is a possibility that oxidative stress may occur in synovial fluid in acute arthritis [17]. Since comparison were made between different individuals in previous studies $[5,17,21]$, it is possible that differences were due to arthritis or other factors such as diet and exercise level. In the current study, the differences were confirmed in the same individual, suggesting that oxidative stress can occur in synovial fluid during arthritis in the horse.

Although it performed measurements in separate individuals, a previous study showed a decrease in antioxidant capacity in acute cases [17]. Since the results of the present study showed similar results in the same individual, it can be said that the antioxidant capacity of synovial fluid decreases in acute cases. This study performed measurements in an acute case, so a decrease in antioxidant capacity was observed. However, if the measurements were carried out in a chronic case, antioxidant capacity may increase due to the adaptive response $[17,21]$. Therefore, in order to evaluate the oxidative stress state of synovial fluid, it is necessary to consider the period from onset.

In humans, it was suggested that oxidative stress in serum was related to the severity of osteoarthritis [6]. However, since oxidative stress in serum was affected by inflammation of other sites of the joint, oxidative stress in synovial fluid is thought to more accurately reflect the condition of the joint than oxidative stress in serum [7]. Therefore, it was considered that measuring the oxidative stress in the synovial fluid of a horse may be useful for determining the severity of arthritis in horses.

In humans, reducing oxidative stress in patients with osteoarthritis has been reported to be effective for improvement of quality of life and physical performance by reducing oxidative protein damage [16]. Oxidative stress generated during arthritis is thought to cause oxidative damage in synovial fluid protein and cartilage matrix, and death of chondrocytes. Thus, oxidative stress is considered to enhance destruction of the joint structure. Indeed, the possibility that destruction of cartilage matrix occurred through oxidative stress was shown in horses [21]. Improvement of oxidative stress is thought to be useful for preservation of joint structure in situations in which oxidative stress is generated in the joint. Therefore, reducing oxidative stress in horses with arthritis may be useful for arthritis treatment. A reduction in oxidative stress may suppress the production of ROS and/or improve antioxidant capacity. However, it is difficult to suppress the production of ROS during inflammation. Therefore, to reduce the oxidative stress in the synovial fluid during arthritis, it is important to improve the antioxidant capacity. At early stages of arthritis, oxidative stress can be reduced by intra-articular administration of substances having antioxidant capacity, such as vitamin $\mathrm{C}$, vitamin $\mathrm{E}$, astaxanthin, and low molecular weight hyaluronic acid [11, 19, 23].

This study revealed that oxidative stress develops in the synovial fluid of horses during arthritis due to carpal joint fracture. Oxidative stress may damage the articular cartilage; therefore, oxidative stress generated in the synovial fluid during arthritis may damage articular cartilage present at sites other than the injured site. Future studies should evaluate the effect of reducing oxidative stress in synovial fluid for arthritis treatment.

\section{References}

1. Angthong, C., Morales, N.P., Sutipornpalangkul, W., Khadsongkram, A., Pinsornsak, P., and Pongcharoen, B. 2013. Can levels of antioxidants in synovial fluid predict the severity of primary knee osteoarthritis: a preliminary study. Springerplus 2: 652. [Medline] [CrossRef]

2. Benbarek, H., Grülke, S., Deby-Dupont, G., Deby, C., Mathy-Hartert, M., Caudron, I., Dessy-Doize, C., Lamy, M., and Serteyn, D. 2000. Cytotoxicity of stimulated equine neutrophils on equine endothelial cells in culture. Equine Vet. J. 32: 327-333. [Medline] [CrossRef]

3. Celi, P., Sullivan, M., and Evans, D. 2010. The stability of the reactive oxygen metabolites (d-ROMs) and biological antioxidant potential (BAP) tests on stored horse blood. Vet. J. 183: 217-218. [Medline] [CrossRef]

4. Chen, K.C., and Chang, L.S. 2009. Arachidonic acid-induced apoptosis of human neuroblastoma SK-N-SH cells is mediated through mitochondrial alteration elicited by ROS and $\mathrm{Ca}^{2+}$-evoked activation of p38alpha MAPK and JNK1. Toxicology 262: 199-206. [Medline] [CrossRef]

5. Dimock, A.N., Siciliano, P.D., and McIlwraith, C.W. 2000. Evidence supporting an increased presence of reactive oxygen species in the diseased equine joint. Equine Vet. $J$. 32: 439-443. [Medline] [CrossRef]

6. Ertürk, C., Altay, M.A., Bilge, A., and Çelik, H. 2017. Is there a relationship between serum ox-LDL, oxidative stress, and PON1 in knee osteoarthritis? Clin. Rheumatol. 36: 2775-2780. [Medline] [CrossRef]

7. Firuzi, O., Spadaro, A., Spadaro, C., Riccieri, V., Petrucci, R., Marrosu, G., and Saso, L. 2008. Protein oxidation markers in the serum and synovial fluid of psoriatic ar- 
thritis patients. J. Clin. Lab. Anal. 22: 210-215. [Medline] [CrossRef]

8. Franck, T., Kohnen, S., de la Rebière, G., Deby-Dupont, G., Deby, C., Niesten, A., and Serteyn, D. 2009. Activation of equine neutrophils by phorbol myristate acetate or $\mathrm{N}$ formyl-methionyl-leucyl-phenylalanine induces a different response in reactive oxygen species production and release of active myeloperoxidase. Vet. Immunol. Immunopathol. 130: 243-250. [Medline] [CrossRef]

9. Güven, O., Tekin, U.S., Durak, I., Keller, E.E., and Hatipoglu, M. 2007. Superoxide dismutase activity in synovial fluids in patients with temporomandibular joint internal derangement. J. Oral Maxillofac. Surg. 65: 1940-1943. [Medline] [CrossRef]

10. Ishimaru, K., Ohba, S., Yoshimura, H., Matsuda, S., Ishimaru, J., and Sano, K. 2015. Antioxidant capacity of synovial fluid in the temporomandibular joint correlated with radiological morphology of temporomandibular disorders. Br. J. Oral Maxillofac. Surg. 53: 114-120. [Medline] [CrossRef]

11. Ke, C., Sun, L., Qiao, D., Wang, D., and Zeng, X. 2011. Antioxidant acitivity of low molecular weight hyaluronic acid. Food Chem. Toxicol. 49: 2670-2675. [Medline] [CrossRef]

12. Kinnunen, S., Atalay, M., Hyyppä, S., Lehmuskero, A., Hänninen, O., and Oksala, N. 2005. Effects of prolonged exercise on oxidative stress and antioxidant defense in endurance horse. J. Sports Sci. Med. 4: 415-421. [Medline]

13. Kirschvink, N., de Moffarts, B., and Lekeux, P. 2008. The oxidant/antioxidant equilibrium in horses. Vet. J. 177: 178-191. [Medline] [CrossRef]

14. Kusano, K., Yamazaki, M., Kiuchi, M., Kaneko, K., and Koyama, K. 2016. Reference range of blood biomarkers for oxidative stress in Thoroughbred racehorses (2-5 years old). J. Equine Sci. 27: 125-129. [Medline] [CrossRef]

15. Manunta, A.F., Zedde, P., Cudoni, S., Caggiari, G., and Pintus, G. 2016. Early joint degeneration and antagonism between growth factors and reactive oxygen species. Is non-surgical management possible? Joints 3: 123-128.
[Medline] [CrossRef]

16. Manoy, P., Yuktanandana, P., Tanavalee, A., Anomasiri, W., Ngarmukos, S., Tanpowpong, T., and Honsawek, S. 2017. Vitamin D supplementation improves quality of life and physical performance in osteoarthritis patients. Nutrients 9: E799. [Medline] [CrossRef]

17. Müller, A.J., Letelier, M.E., Galleguillos, M.A., MolinaBerríos, A.E., and Adarmes, H.H. 2010. Comparison of the antioxidant effects of synovial fluid from equine metacarpophalangeal joints with those of hyaluronic acid and chondroitin sulfate. Am. J. Vet. Res. 71: 399-404. [Medline] [CrossRef]

18. Po, E., Williams, C., Muscatello, G., and Celi, P. 2013. Assessment of oxidative stress biomarkers in exhaled breath condensate and blood of Thoroughbred foals. Vet. J. 196: 269-271. [Medline] [CrossRef]

19. Sato, F., Omura, T., Ishimaru, M., Endo, Y., Murase, H., and Yamashita, E. 2015. Effects of daily astaxanthin and 1-carnitine supplementation for exercise-induced muscle damage in training Thoroughbred horses. J. Equine Vet. Sci. 35: 836-842. [CrossRef]

20. Tsuchiya, M., Sato, E.F., Inoue, M., and Asada, A. 2008. Open abdominal surgery increases intraoperative oxidative stress: can it be prevented? Anesth. Analg. 107: 1946-1952. [Medline] [CrossRef]

21. Villasante, A., Araneda, O.F., Behn, C., Galleguillos, M., and Adarmes, H. 2010. Antioxidant capacity and oxidative damage determination in synovial fluid of chronically damaged equine metacarpophalangeal joint. Vet. Res. Commun. 34: 133-141. [Medline] [CrossRef]

22. Williams, C.A. 2016. The effect of oxidative stress during exercise in the horse. J. Anim. Sci. 94: 4067-4075. [Medline] [CrossRef]

23. Wu, Q., Wang, X., Nepovimova, E., Wang, Y., Yang, H., Li, L., Zhang, X., and Kuca, K. 2017. Antioxidant agents against trichothecenes: new hints for oxidative stress treatment. Oncotarget 8: 110708-110726. [Medline] [CrossRef] 